\title{
Diseño e implementación de un Sistema de adquisición y monitoreo de datos (SHM) para un rectificador de protección catódica usado en ductos.
}

\section{Design and implementation of an acquisition and data monitoring system (SHM) for a cathodic protection rectifier used in pipelines.}

\section{Desenho e implementação de um Sistema de aquisição e monitoramento de dados (SHM) para um retificador de proteção catódica utilizado em condutas.}

\author{
Daniel Alejandro Rodríguez-Caro ${ }^{1}$, Enrique Vera-López², Helver Mauricio Muñoz-Barajas ${ }^{3}$
}

Forma de citar: D. A. Rodríguez-Caro, E. Vera-López, H. M. Muñoz-Barajas, "Diseño e implementación de un Sistema de adquisición y monitoreo de datos (SHM) para un rectificador de protección catódica usado en ductos", Respuestas, vol. 21, no. 1, pp. 45-55, 2016.

Recibido:

Mayo 26 de 2015

Aceptado:

Agosto 19 de 2015

\section{Resumen}

Antecedentes: La protección catódica por corriente impresa es uno de los métodos para prevenir la corrosión de tuberías o tanques, preservando el estado estructural y la integridad del material. Para que un sistema de protección catódica funcione correctamente debe existir un control sobre las variables eléctricas que intervienen en el proceso, es por ello que se hace necesario monitorear variables tales como (Voltaje, Corriente y Potencial de protección). Objetivo: De esta manera se desarrolla un sistema de adquisición y monitoreo de datos en tiempo real, con el propósito de aumentar la accesibilidad a las variables eléctricas y de esta forma mejorar el funcionamiento del sistema de protección catódica. Métodos: El sistema de monitoreo y análisis de la información se basa en el concepto de SHM (Structural Health Monitoring), el cual consta de; un sistema electrónico de adquisición y envío remoto de señales (micro controlador y sistema GSM de comunicaciones) y un sistema de visualización y análisis de la información en un sistema móvil (celular), usando un servidor web para ello. Teniendo en cuenta que la condición de integridad estructural del ducto está determinada por el correcto funcionamiento del rectificador. Resultados: se logró implementar un sistema de monitoreo y visualización remota de las variables principales de un sistema de protección catódica. Se desarrolló un algoritmo basado en el concepto de SHM, el cual permite correlacionar, generar tendencia y establecer criterios de funcionamiento del sistema de protección catódica que permiten establecer si el sistema está asegurando la integridad estructural del ducto de transporte de crudo. Conclusión: lo novedoso del presente trabajo consiste en mostrar el comportamiento en tiempo real de las variables necesarias para analizar si el ducto está siendo correctamente protegido y generar las alarmas e informes sobre protección catódica, lo cual es la base del concepto de SHM (Structural Health Monitoring).

Palabras Clave: corriente, corrosión, Innovación, monitoreo, SHM (Structural Health Monitoring).

\section{Abstract:}

Background: Cathodic protection by impressed current is one of the methods to prevent corrosion of pipes or tanks, preserving the structural state and integrity of the material.
${ }^{3}$ Ingeniero Mecánico orcid:0000-0002-5298-3315

Universidad Pedagógica y Tecnológica de Colombia Tunja - Colombia 
No. 1

Enero - Junio 2016 ISSN 0122-820X E-ISSN 2422-5053

For a cathodic protection system to function properly there must to be a control over the electrical variables involved in the process, which is why it is necessary to monitor variables such as (voltage, current and potential protection). Objective: to develop a system of data acquisition and monitoring in real time, in order to increase accessibility to electrical variables and thus improve the operation of the cathodic protection system. Methods: The monitoring and information analysis system is based on the concept of SHM (Structural Health Monitoring), which consists of an electronic system for remote acquisition and sending of signals (micro controller and GSM communications system) and a system for visualization and analysis of information in a mobile system (cell) using a web server for it. Given that the condition of structural integrity of the pipeline is determined by the correct operation of the rectifier. Results: It was possible to implement a monitoring and remote viewing system of the main variables of a cathodic protection system. An algorithm based on the concept of SHM was developed, allowing to correlate, generate trend and establish performance criteria for the cathodic protection system which allows to establish whether the system is ensuring the structural integrity of the crude transportation pipeline. Conclusion: the novelty of this work is to show the realtime behavior of the variables needed to analyze whether the pipeline is being properly protected and generate alarms and reports regarding cathodic protection, which is based on the concept of SHM (Structural Health Monitoring).

Keywords: Current, Corrosion, Index Terms - Innovation, monitoring, SHM (Structural Health Monitoring).

\section{Resumo}

Antecedentes: A proteção catódica por corrente impressa é um dos métodos para prevenir a corrosão do transporte tubular ou condutas, preservando o estado estrutural e a integridade do material. Para que um sistema de proteção catódica funcione corretamente deve existir um controle sobre as variáveis eléctricas que estão envolvidas no processo, é por isso que é necessário monitorar variáveis tais como: voltagem, corrente e potencial de proteção. Objetivo: Desenvolver um sistema de aquisição e monitoramento de dados em tempo real, com o propósito de aumentar a acessibilidade às variáveis eléctricas e desta maneira melhorar o funcionamento do sistema de proteção catódica. Métodos: O sistema de monitoramento e análise da informação se baseia no concepto de SHM (Structural Health Monitoring), que compreende um sistema electrónico de aquisição e envio remoto de sinais (micro controlador e sistema GSM de comunicações) e um sistema de visualização e análise da informação num sistema móvel (celular), usando para isto um servidor web. Tendo em conta que a condição de integridade estrutural do transporte tubular está determinada pelo correto funcionamento do retificador. Resultados: Foi possível implementar um sistema de monitorização e visualização remota das variáveis principais de um sistema de proteção catódica. Foi desenvolvido um algoritmo baseado no conceito de SHM, que permite correlacionar, gerar tendência e estabelecer critérios de desempenho para o sistema de proteção catódica, permitindo estabelecer se o sistema garante a integridade estrutural da tubulação de transporte de bruto. Conclusão: A novidade deste trabalho é mostrar o comportamento em tempo real das variáveis necessárias para analisar se o transporte tubular está sendo corretamente protegido e gerar as alarmes e relatórios sobre a proteção catódica, que se baseia no conceito de SHM (Structural Health Monitoring).

Palavras-chave: corrente, corrosão, inovação, monitoramento, SHM (Structural Health Monitoring). 


\section{Introducción}

En la industria petrolera, el transporte de petróleo se realiza usando los Oleoductos, los cuales son ductos enterrados que poseen dos tipos de protección contra la corrosión generada por los suelos principalmente; recubrimientos y protección catódica.

La protección catódica es un sistema que imprime corriente al ducto, compuesto principalmente por un rectificador de protección catódica y unos elementos complementarios como son: las camas anódicas, estaciones de monitoreo, etc. En este aspecto dicha protección polariza la estructura a un potencial negativo, garantizando suficiente carga para que se mantenga la integridad estructural de la estructura (ducto o tanque) en caso de daños en el revestimiento.

Cuando se polariza negativamente la estructura, ésta adquiere un potencial, el cual es medido respecto a un electrodo de referencia (normalmente $\mathrm{Cu} / \mathrm{CuSO}_{4}$ ) [1], [2]. Existen criterios internacionales [3], [4], que determinan que el potencial del protección del ducto debe no ser más positivo que $-0.85 \mathrm{~V}$, ni más negativo que $-1.4 \mathrm{~V}$ (todo esto, respecto al electrodo de referencia $\mathrm{Cu} / \mathrm{CUSO}_{4}$ ). Potenciales de protección más negativos que $-1.4 \mathrm{~V}$, podrían generar problemas en el recubrimiento, induciendo un desprendimiento catódico "disbonding catódico" y por ende afectando la integridad del ducto o tanque.

Existen tres parámetros que se deben monitorear, con el fin de establecer un buen funcionamiento de un sistema de protección catódica: el voltaje de salida del rectificador, la corriente de salida del rectificador y el potencial de protección medido respecto a un electrodo de referencia (en el caso de ducto, este se mide a lo largo del mismo).

Por esta razón, se hace muy importe monitorear de manera continua los valores del potencial de protección del ducto que de una u otra manera está relacionado con los valores de voltaje y corriente de salida del rectificador. Si existe una desviación de estos valores antes mencionados, existe el riesgo que el ducto no esté protegido y ocurran problemas graves de corrosión (potenciales más positivos de $-0.85 \mathrm{~V}$ $\mathrm{Cu} / \mathrm{CuSO}_{4}$ ) o problemas de desprendimiento del recubrimiento (disbonding catódico), que se presentarían por la aplicación de sobrepotenciales de protección (más negativos de $\left.-1.4 \mathrm{~V} \mathrm{Cu} / \mathrm{CuSO}_{4}\right)$.

Teniendo en cuenta lo anteriormente expuesto, es muy importante poder tener información acertada y en tiempo real del funcionamiento de los rectificadores y las variables que se manejan en estos sistemas de protección catódica. En Colombia existen más de 2000 rectificadores instalados, que son de funcionamiento manual, lo que significa que el personal encargado de hacer seguimiento a la protección catódica deba movilizarse a los sitios remotos donde están instalados estos equipos para verificar las variables.

En este estudio se implementó un sistema de monitoreo y análisis de las variables en tiempo real. Su implementación constó de varias etapas: construcción de un sistema de acondicionamiento, adquisición y envío de la información a un servidor web remoto (Se usó la implementación de un micro-controlador acoplado un sistema GSM) y un sistema de visualización y análisis de la información basado en plataformas móviles (visualización en celular), el cual permitió verificar las variables en el tiempo y generar las alarmas correspondientes para generar los planes de acción (concepto SHM, monitoreo de las variables que aseguran la integridad mecánica de las estructuras -caso de los ductos). [5]

Se usó un micro controlador ATmega328 para el acondicionamiento y monitoreo de las variables eléctricas del sistema de protección catódica. Las variables fueron almacenadas en una memoria SD para poder llevar un registro propio en el módulo, enviar los datos por comunicación GSM/GPRS a través de un módulo Quectel M95, haciendo uso del
Enero - Junio 2016 ISSN 0122-820X E-ISSN 2422-5053 PP: $45-55$ 
No. 1

Enero - Junio 2016 ISSN 0122-820X E-ISSN 2422-5053

PP: 45-55 protocolo HTTP y adicionalmente transmitir la información al servidor que incluye tanto la Base de Datos como los registros.

El sistema de análisis y visualización de la información fue desplazada en un dispositivo móvil (celular o página web), que accede al servidor WEB y que mantiene la base de datos.

\section{Materiales y Métodos}

En la presente sección se describe la metodología desarrollada, la cual se llevó a cabo en dos etapas principales:

- Desarrollo del sistema de adquisición y algoritmo de transmisión de datos basados en GSM, de las tres variables de un sistema de protección catódica (voltaje y corriente de salida del rectificador) y voltaje de protección del ducto, referenciado respecto al electrodo de $\mathrm{Cu} / \mathrm{CUSO}_{4}$.

- Desarrollo de una metodología de evaluación de estas variables, tal que se puedan generar correlaciones, tendencias y determinar el buen funcionamiento de la protección catódica y así asegurar la integridad mecánica de la tubería, especialmente asegurando la protección contra la corrosión. Este aspecto es el relevante en el concepto de SHM, el cual aplica para asegurar condiciones de operación óptimas sin fallas que afecten la integridad y seguridad del ducto.

\subsection{Rectificador de protección catódica.}

48 En sistemas de protección catódica por corriente impresa es necesario tener un rectificador que en términos generales se encarga de transformar la corriente alterna en corriente directa, permitiendo un control en el flujo de electrones y así el proceso electroquímico de protección se lleve a cabo en los oleoductos. Un rectificador de protección catódica se caracteriza principalmente por sus valores de voltaje y corriente. Normalmente se utilizan fuentes de voltaje, de tal manera que para un voltaje determinado la corriente está controlada por la resistencia, que en el caso de ducto es la resistencia total del circuito (principalmente la resistencia de la cama anódica).

Así, la función del rectificador de protección catódica es la de suministrar corriente directa a la estructura (ducto) de tal manera que polarice negativamente la estructura y de esta manera se garantice que el acero del ducto no va a tener problemas de corrosión.

Además el sistema rectificador cuenta con diversos componentes de protección contra sobretensiones o posibles daños por corto circuito junto a una resistencia "Shunt" que permite obtener medidas de altas corrientes en la salida del rectificador.

Cerca al rectificador y en un punto cercano al ducto, se instala (enterrado) un electrodo de referencia fijo $\left(\mathrm{Cu} / \mathrm{CuSO}_{4}\right)$, el cual sirve para medir el potencial de protección del ducto. El diagrama esquemático de un rectificador para protección catódica se puede observar en la Figura 1, donde se pueden apreciar los puntos de medición de variables eléctricas (Voltaje de referencia (V1), Voltaje de Salida (V2) y Corriente de Salida (V3). La corriente de salida se calcula como la caída de voltaje en una resistencia resistencia "shunt".

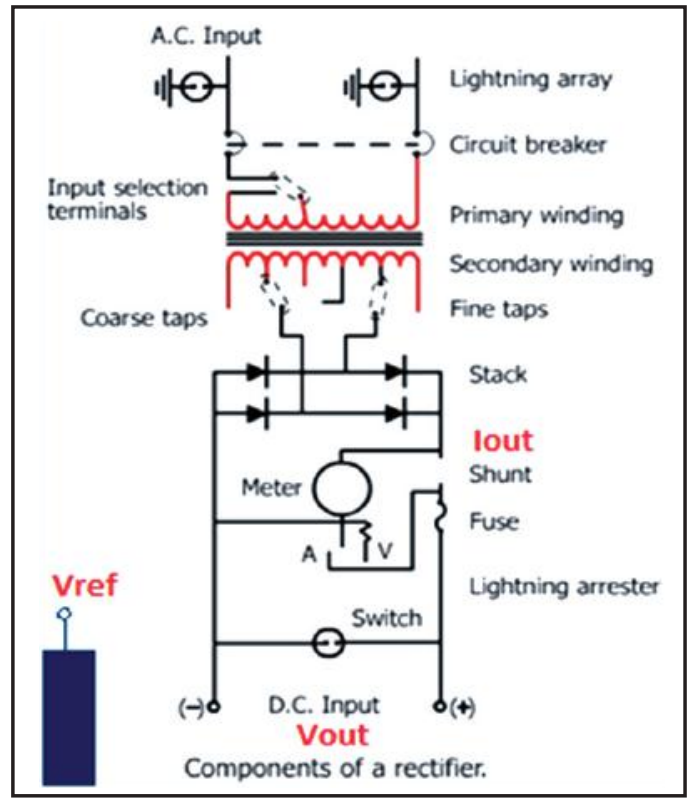

Figura 1. Esquemático de un rectificador para ICCP Fuente: [6] 
En la figura 2, se presenta un esquema general de un sistema de protección catódica para la protección de un ducto. En este esquema, se pueden observar las variables que va a ser monitoreadas (V1, V2 y V3).

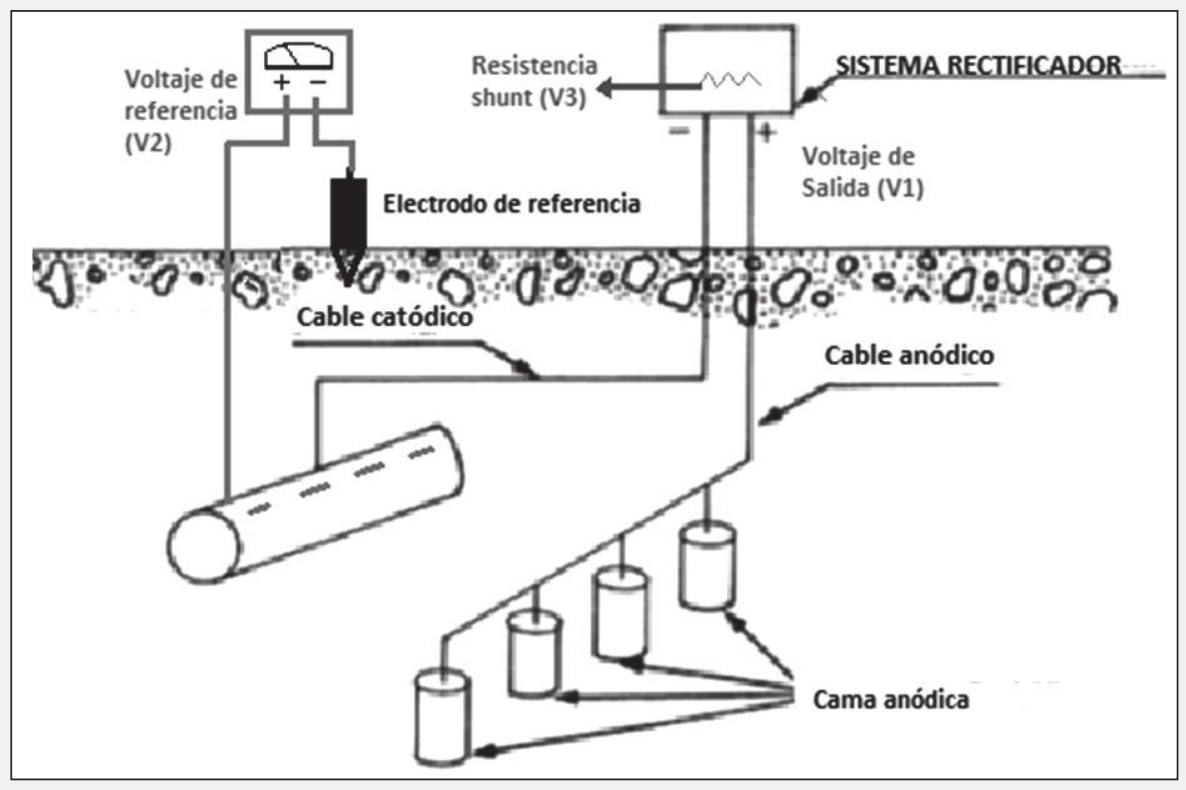

Figura 2. Sistema general de protección catódica por corriente impresa para ductos. Fuente: [7]

V1 y V3, son las variables del rectificador (voltaje de salida del rectificador y corriente de salida del rectificador). Como se observa en esta figura la corriente se toma como la caída de voltaje en una resistencia "Shunt", que posee el rectificador.

El voltaje V2, es el voltaje de protección del ducto (nos determina que tan polarizado está el ducto) el cual está medido respecto a un electrodo de referencia, el cual normalmente es el de Cobre - Sulfato de Cobre $\left(\mathrm{Cu} / \mathrm{SO}_{4}\right)$.

Los cambios que puedan tener cada una de estas variables son vitales para interpretar la efectividad en el control de corrosión e integridad estructural del ducto; por ejemplo un aumento en la corriente del rectificador, podría ser un indicativo que el revestimiento del ducto se está deteriorando o la resistencia del suelo ha disminuido (época de lluvias), lo cual llevaría a ejercer cambios en las condiciones del mismo.

\subsection{Sistema de adquisición y acondicionamiento de señales}

Para adquirir los datos de corriente y voltaje de salida como también el voltaje de referencia en protección catódica, es necesario acondicionar las señales para que el sistema micro-controlador pueda trabajar correctamente la conversión análoga digital (ADC), haciendo que la adquisición de voltajes se encuentre en un rango de $0 \mathrm{v}$ y $5 \mathrm{v}$.

Cuando los valores de voltaje son mayores al rango de trabajo del micro-controlador, se debe realizar un divisor de tensión donde se determina la relación de voltajes altos y bajos. Fue necesario usar amplificadores de instrumentación, tal que los voltajes de la variables (V1, V2 y V3) ingresaran al ADC del micro-controlador "arduino"[8] ATmega328 el cual posee una resolución del conversor análogo digital de 10 bits, así se obtienen valores procesados en bits o en formato digital, usando la fórmula de traspaso al valor real descrita en la "ecuación (1)", donde FC 
No. 1

Enero - Junio 2016 ISSN 0122-820X E-ISSN 2422-5053 PP: 45-55 es el valor calculado para cada variable a medir, $\mathbf{V}$ digital es el dato adquirido después de la conversión digital.

Valor $=\left(\frac{5(\text { Vdigital })}{1023}\right) * F C$

De esta manera obtener un empleo más simple de los datos en sistemas de procesamiento y comunicación.

En la Figura 3 se muestra el esquema general del acondicionamiento de las señales que ingresan al "Arduino uno" (Atmega328).

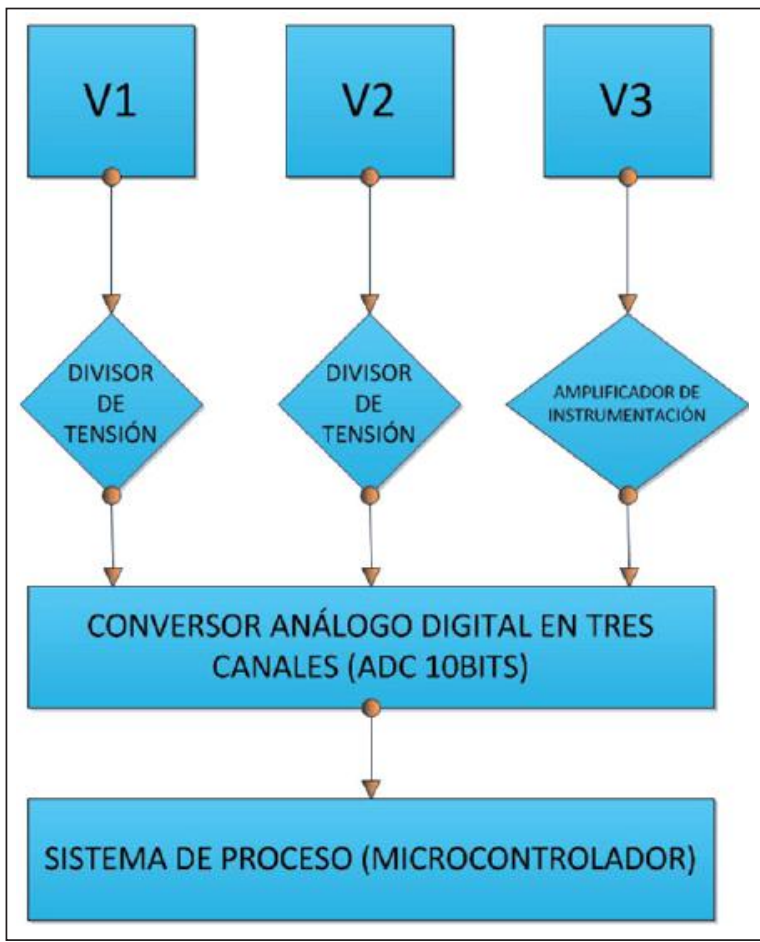

Figura 3. Acondicionamiento de las señales Fuente: Autores. módulo Quectel M95, que junto con una SIMcard con plan de navegación de internet, envía información desde la red celular a la red mayor (internet).

En la Figura 4, se presenta el esquema general de comunicaciones.

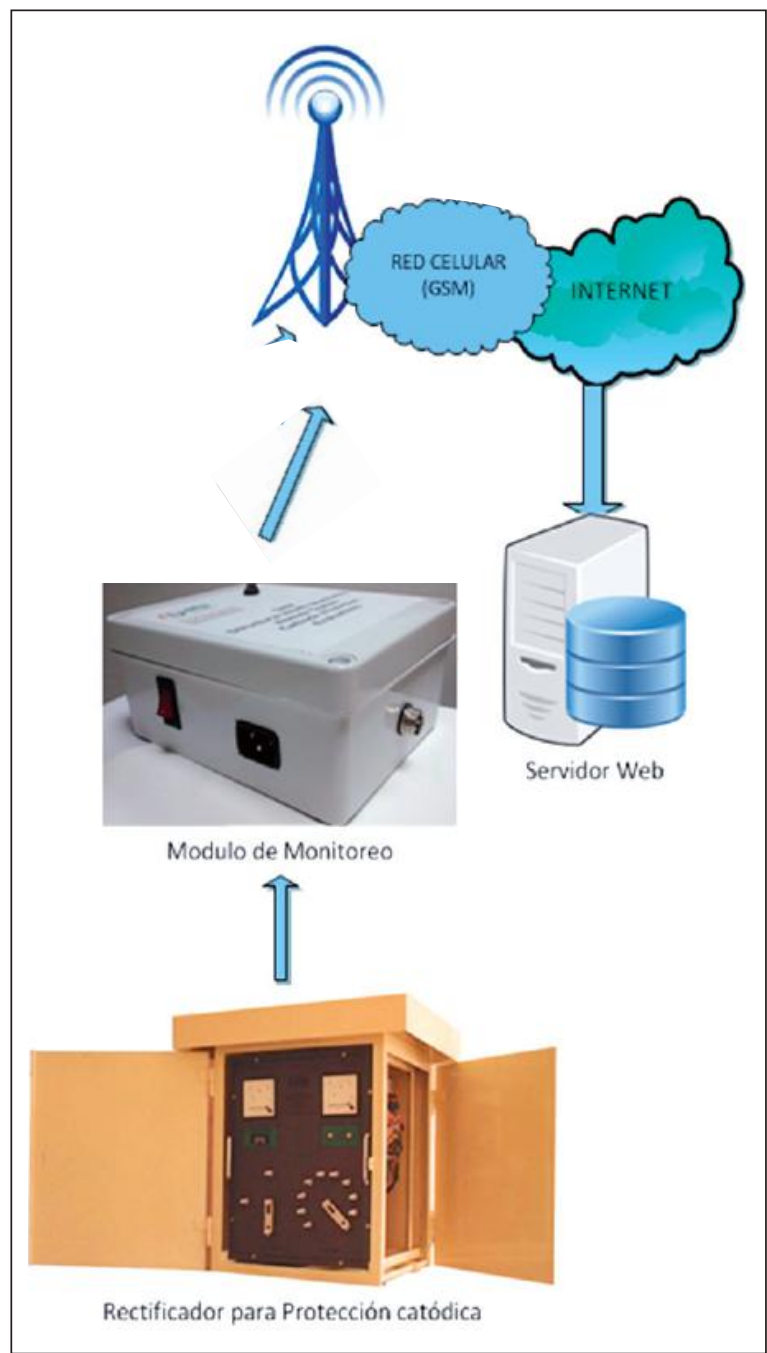

Figura 4. Mapa de comunicación del sistema de monitoreo Fuente: Autores.

En la figura 5, se puede observar de manera específica, el sistema de almacenamiento de los datos. El cual usa un "datalogger", permitiendo guardar los datos en una memoria SD para un registro interno y así asegurar la medición de las variables en el equipo de monitoreo sin importar fallos en la red GSM/ GPRS. (Comunicación usando un Quectel M95 y protocolo HTTP). En general la figura 5 , muestra el concepto de telemetría usado en la investigación.

Para lograr dicho proceso se utiliza un 


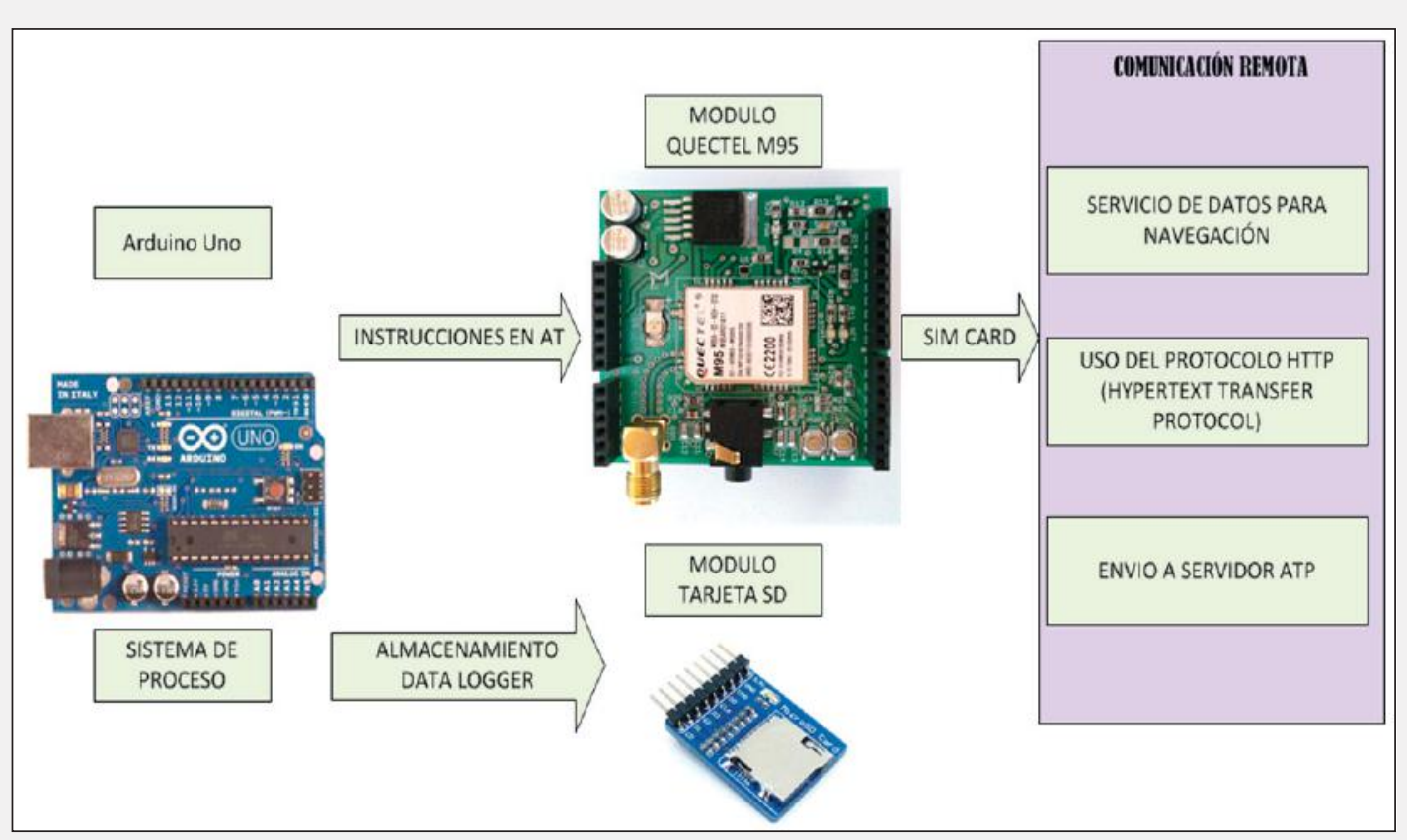

No. 1

Enero - Junio 2016

ISSN 0122-820X

E-ISSN 2422-5053

PP: 45-55

Figura 5. Comunicación y almacenamiento Fuente: Autores.

\subsection{Análisis de información - Implementación de sistema SHM}

El concepto de visualización y análisis de la información, se basa en la metodología SHM (Structural Health Monitoring), la cual busca generar la adecuada interpretación, correlación de variables y generación de tendencia, principalmente basados en criterios y estándares internacionales, que fijan los niveles de aceptación de cada una de las variables que se analizan. Par el caso de esta investigación, las variables monitoreadas (Voltaje y corriente del rectificador; V1 y V3) permiten establecer la condiciones del revestimiento del ducto, las condiciones de humedad del suelo y por ende de los límites de funcionamiento del rectificador. Por otra parte el potencial de protección (V3), establece los criterios de la adecuada protección y su interpretación permite establecer si la corriente impresa al ducto es la adecuada para garantizar la integridad estructural del mismo. Inadecuados valores de V3, pueden llevar a que la integridad del ducto sea afectada por la corrosión o que se generan problemas de desprendimiento catódico del revestimiento, lo cual afectaría el concepto de "salud estructural del ducto".
En general el concepto SHM, es una metodología, usada para evaluar el estado en que se encuentra un equipo (ductos) frente a morfologías y cambios en su composición integral. Así mismo, poder determinar los síntomas y causas para proponer un diagnóstico que evite la repetición de fallas en el caso particular, las tuberías enterradas y a la vez aumenta el índice de mantenimiento predictivo que el correctivo.

También se proyecta obtener una mejora en los tiempos de respuesta frente a la identificación de alguna falla en la integridad del material del oleoducto, llegando a tal punto que en el estado integral del material no exista ninguna clase de accidente por causa de la corrosión del material o desprendimiento catódico del revestimiento del ducto.

\section{Resultado y análisis}

3.1 Módulo de acondicionamiento y adquisición de datos con comunicación GSM/GPRS:

Como resultados, se obtiene un módulo de acondicionamiento y adquisición de datos para sistemas de protección catódica por corriente impresa con comunicación GSM/ 
Enero - Junio 2016

ISSN 0122-820X

E-ISSN 2422-5053

PP: $45-55$
GPRS para el envío de la información con respecto a las variables eléctricas presentes en el rectificador, las cuales trabajan con una resolución de 10 bits en su adquisición y un sistema de RTC ("real time clock") para sincronizar las acciones que el microcontrolador ejecute.

Los datos procesados por el microcontrolador son almacenados en una memoria SD para registrar la información, asimismo conseguir valores confiables para identificar algún problema en el sistema de monitoreo de protección catódica.

Además de su almacenamiento en una memoria interna, el módulo se encarga de enviar datos por medio de la red celular configurando el tiempo de transmisión de datos desde el servidor acorde a la solicitud del usuario, teniendo como limitante, el área de cobertura que disponga el operador donde se encuentra registrada la SIMcard.

En la Figura 6 se puede observar el dispositivo de monitoreo de variables ya instalado en un rectificador para protección catódica de uso en campo.

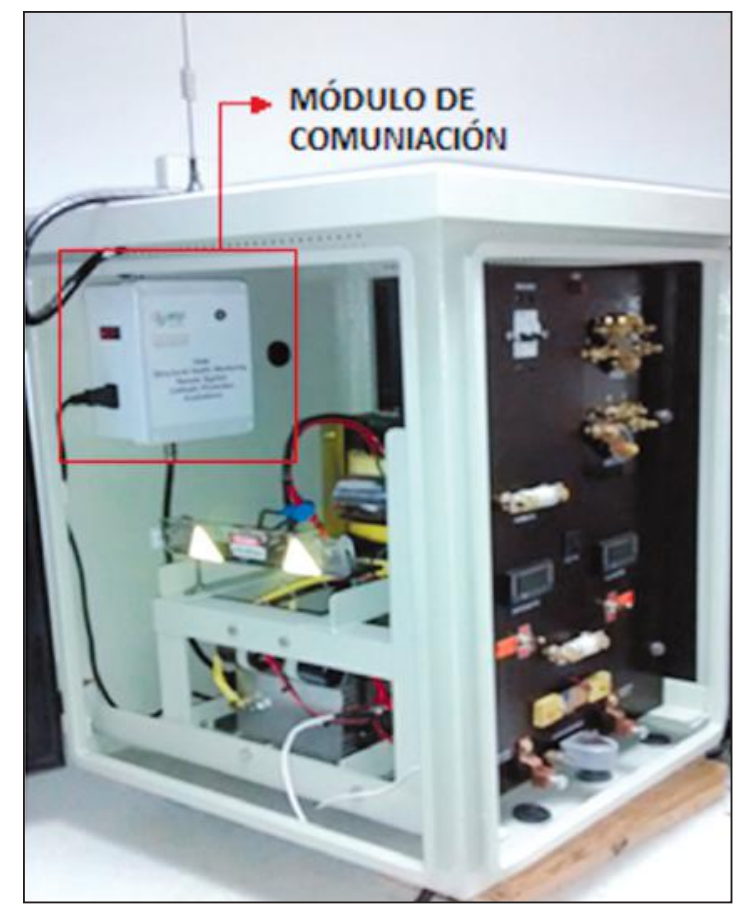

Figura 6. Montaje de módulo de monitoreo en un rectificador Fuente: Autores.

\subsection{Implementación del sistema SHM}

Al implementar el SHM (Structural Health Monitoring) se hace uso de la web 3.0 como herramienta para visualizar y analizar los datos del sistema de monitoreo. Se desarrolla un algoritmo de correlación de variables y análisis de tendencias, tal que permita establecer si el sistema de protección está asegurando la integridad estructural del ducto.

El usuario accede a los resultados de la estudios de información desde cualquier lugar con acceso a la página web (empresa ATP ingeniería) conocida como "GIAP".

En la Figura 7 se puede observar la manera como la información se presenta ante el cliente, identificando la posición geográfica del rectificador haciendo uso de sistemas de posicionamiento global. (Caso de un rectificador localizado en el municipio de Apiay en el Meta - Colombia). 


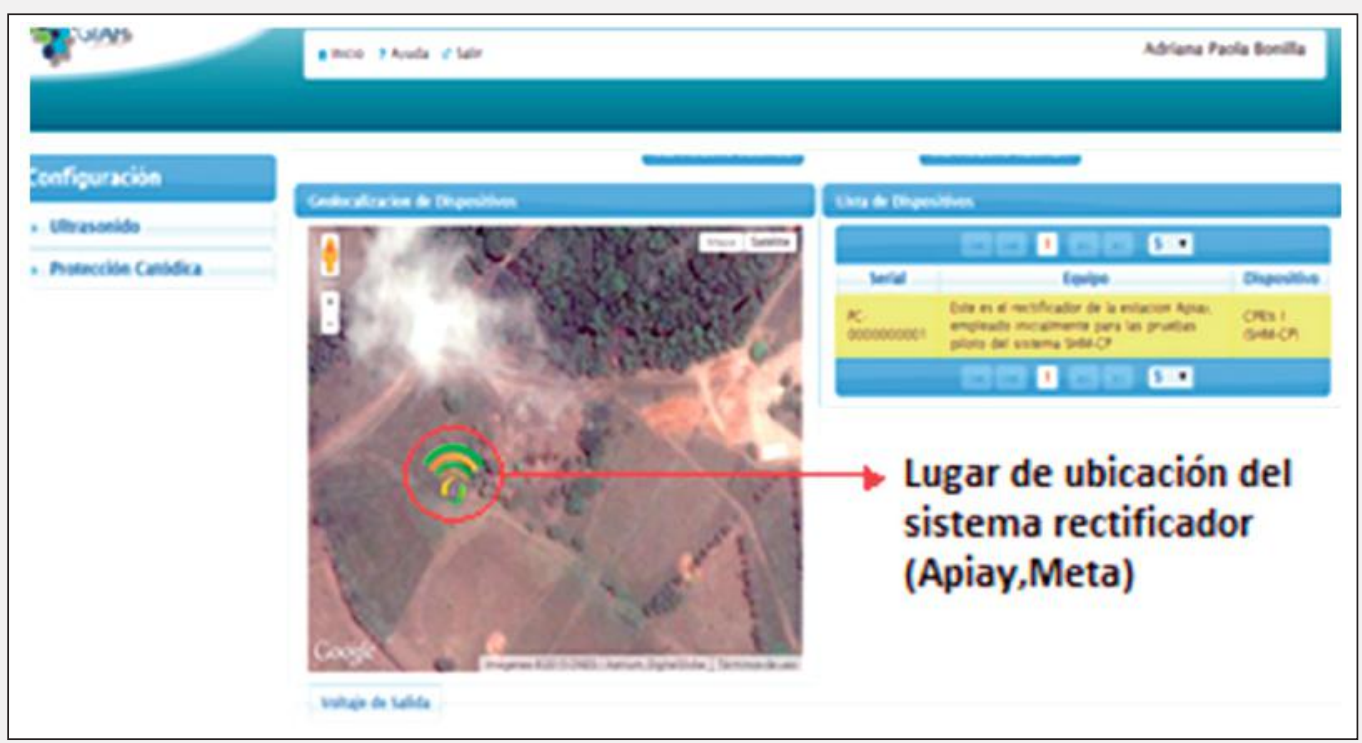

No. 1

Enero - Junio 2016 ISSN 0122-820X

E-ISSN 2422-5053

Figura 7. Sistema de visualización de posicionamiento global e identificación d dispositivo de monitoreo Fuente: Autores.

Se observa que existe una correlación de las variables V1, V2 y V3, tal que se establecen criterios de buen desempeño y/o alarmas que le indican al usuario fallas que estarían afectando la integridad estructural del ducto. Los análisis permiten inferir comportamiento del sistema, tales como cambios en la resistencia del terreno, incremento de las necesidades de corriente debido a fallas del revestimiento y otras.

Un aspecto de gran importancia y que forma parte del concepto SHM, es el análisis de los históricos de comportamiento de las variable, tales que permita inferir tendencias de comportamiento y así establecer las rutinas de control y programas de inspección de equipos, todo esto tendiente a disminuir el riesgo de falla.

La figura 8, muestra una pantalla de visualización vista desde un dispositivo portátil (celular), en la cual se observa el comportamiento de las variables monitoreadas (voltaje respecto al tiempo). Aquí se observa como varia el voltaje de salida del rectificador respecto al potencial de protección del ducto (escala de voltaje de salida del rectificador).

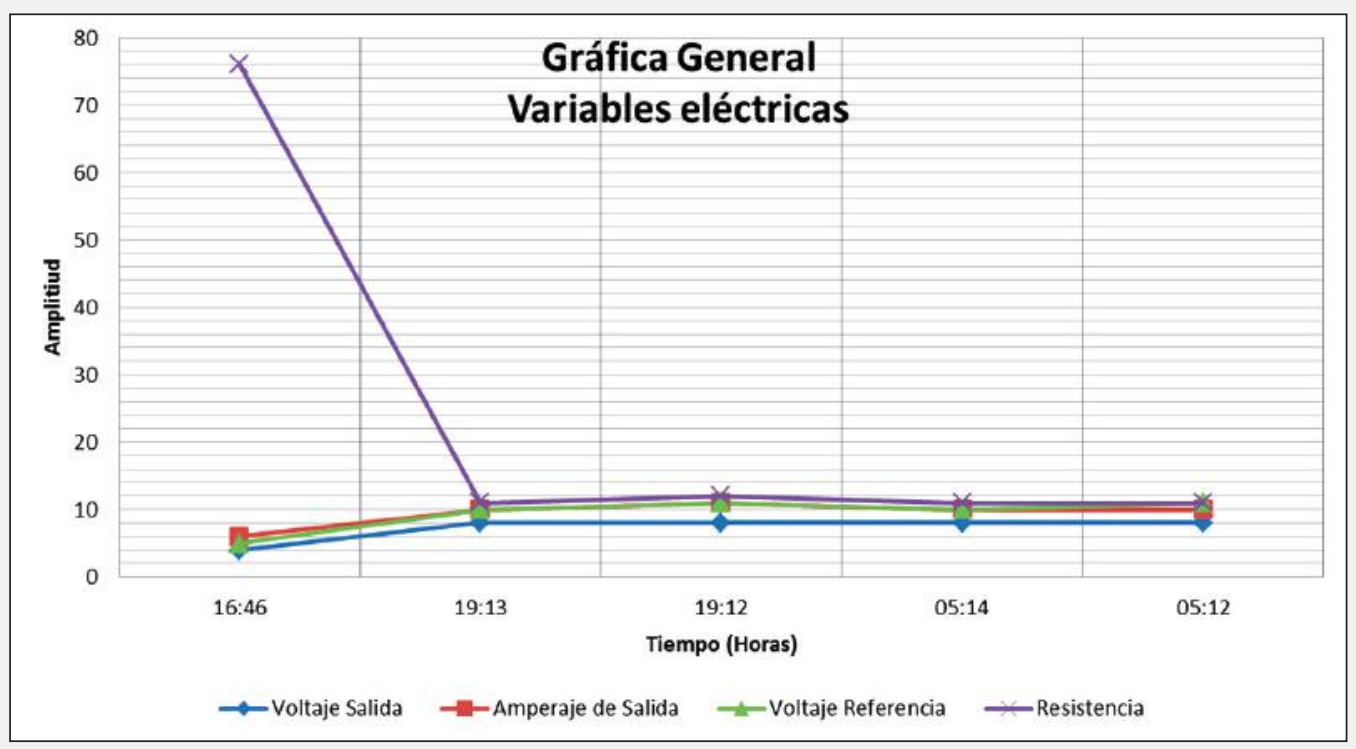

Figura 8. Sistema de visualización de variables y análisis con SHM Fuente: Autores. 
No. 1

Enero - Junio 2016 ISSN 0122-820X E-ISSN 2422-5053 PP: $45-55$
A partir del voltaje de referencia se verifica el funcionamiento del sistema de protección catódica en ducto, el cual debe oscilar entre los $-0,850 \mathrm{~V}$ y $-1,4 \mathrm{~V}$. Valores por fuera de este rango, genera alarmas al usuario, planteándole las posibilidades de acción, como son verificación en campo de estado del rectificador y/o ajuste de los parámetros del mismo.
En la Figura 9, visualiza una gráfica, donde se observa que el sistema dejó de funcionar correctamente. (Valores más positivos que -850 $\mathrm{mV}$ respecto $\mathrm{Cu} / \mathrm{CuSO}_{4}$ ). Este generó una alarma, donde se establece un plan de inspección, el cual identifica que se había caído el suministro de energía del rectificador.

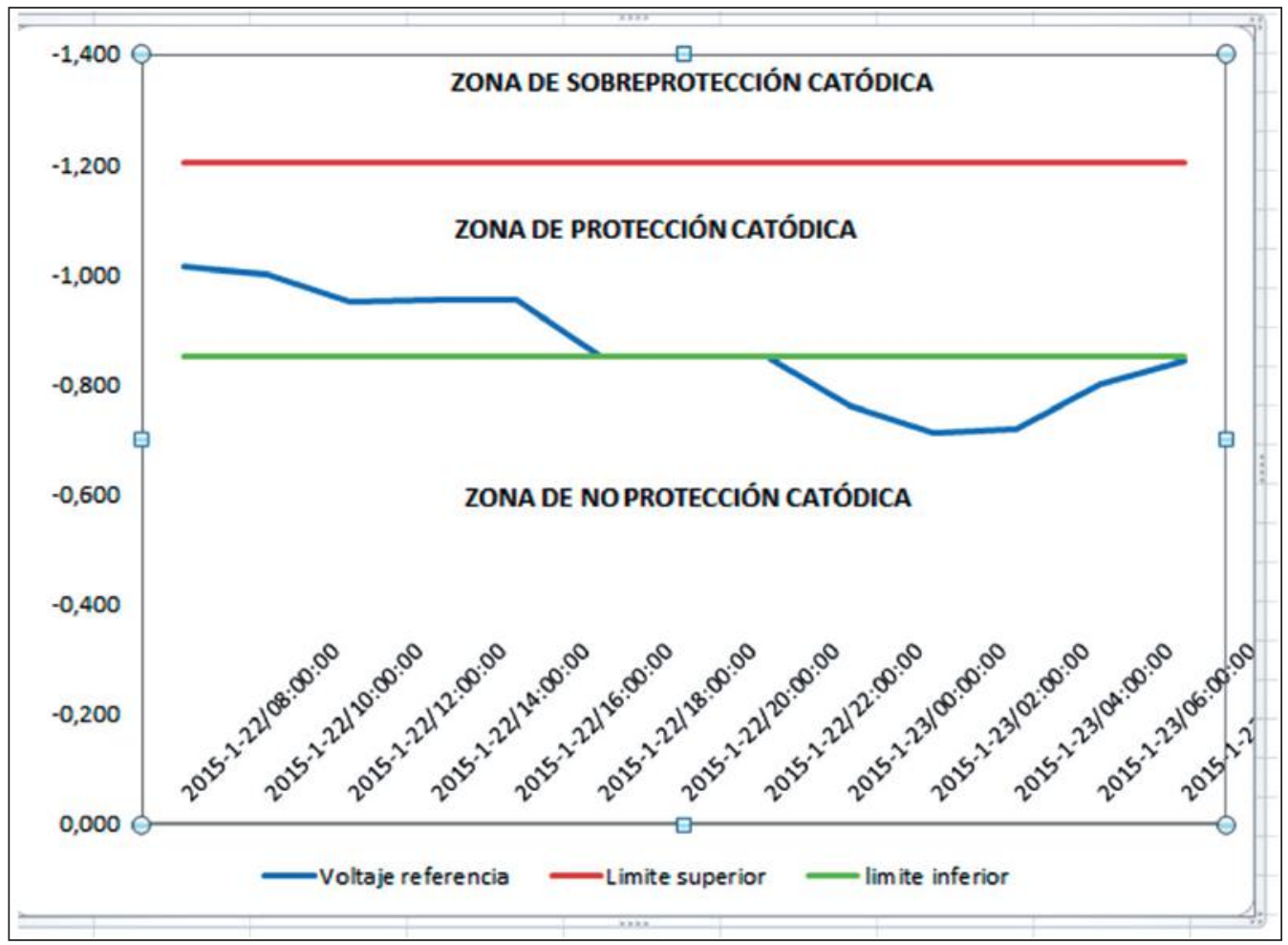

Figura 9. Toma de datos de valor de referencia en un sistema rectificador en Apiay Meta. Fuente: Autores.

Este proyecto, el cual implemento un sistema adecuado de monitoreo y un software de correlación de variables que ante todo se orienta a asegurar la integridad estructural del ducto (caso que se presenta), es una manera adecuada de minimizar el riesgo de falla y asegurar la operación y "core" o función principal de la empresa, que es el transporte de hidrocarburos.

\section{Conclusión}

Los resultados logrados en este proyecto, permitieron implementar un sistema de monitoreo y visualización remota de las variables principales de un sistema de protección catódica. Se desarrolló un algoritmo basado en el concepto de SHM, el cual permite correlacionar, generar tendencia $y$ establecer criterios de funcionamiento del sistema de protección catódica que permiten establecer si el sistema está asegurando la integridad estructural del ducto de transporte de crudo.

El usuario tiene en tiempo real y en un dispositivo móvil la información y análisis de esta, donde visualiza alarmas que se clasifican en función de los planes de acción. Todas las alarmas tienden a que el sistema garantice la integridad y "salud estructural de los ductos", tal que la operación de transporte de curdo 
funcione con riesgos mínimos de falla.

Desde el punto de vista de la electrónica, se usaron componentes de fácil adquisición, implementando un "hardware" y una programación que compite con equipos comerciales en calidad, pero realizados a un menor costo. Desde este punto de vista los resultados del proyecto son innovadores, ya que se creó un dispositivo de desarrollo nacional que puede ser comercializado. El algoritmo SHM implementado en el software de análisis permite consolidar el concepto de la metodología, basada en el concepto de aseguramiento de la integridad estructural de la infraestructura, mediante el monitoreo y análisis de variables y parámetros de un sistema de control de fallas, en este caso un sistema de control de corrosión usando la protección catódica.

\section{Referencias}

[1]. D. A. Jones. "Principles and Prevention of Corrosion", Prentice Hall, Lebanon, Indiana, U.S.A, 1996.

[2]. H. Uhligs. "Corrosión y control de corrosión” 2 ed, Ediciones URNO S.A, España, Madrid, 1.987.

[3]. P. R. Roberge. "Handbook of Corrosion Engineering", Segundo edición, McGraw-Hill, New York, 2012.

[4]. J. H. Morgan. "Cathodic Protection", 2nd ed, NACE International, Houston, United States, 1987.

[5]. S. Gopalakrishnan, M. Ruzzene, and H. Sathyanarayana. "Computational Techniques for Structural Health Monitoring", Springer- Verlag, New York, United States of America, 2011.

[6] M. A. Akcayol, “Application of Fuzzy Logic Controlled Cathodic Protection on Iraq-Turkey Crude Oil Pipeline," Appl. Intell, vol. 24, no. 1, pp. 43-50, Feb. 2006.
[7] S. W. Meier and American Water Works Association. "Steve Meier, Steel Water Storage Tanks: Design, Construction, Maintenance, and Repair", McGrawHill, United, States of America, 2010.

[8]. P. Di Justo, E. Gertz. "Atmospheric Monitoring with Arduino", First Edition O'Reilly Media, United States of America, 2012.

[9]. K. Wesolowski. "Mobile Communication Systems", Wydawnictwa Komunikacji i Lączności sp z o.o., Warszawa, 1.999.
Enero - Junio 2016 ISSN 0122-820X

E-ISSN 2422-5053 PP: 45-55 